\title{
Endocrine deficit after fractionated total body irradiation
}

\author{
A L Ogilvy-Stuart, D J Clark, W H B Wallace, B E Gibson, R F Stevens, S M Shalet, \\ M D C Donaldson
}

\begin{abstract}
Endocrine function was assessed in $\mathbf{3 1}$ children (17 boys) after fractionated total body irradiation used in the preparative regimen for bone marrow transplantation. Endocrine dysfunction was present in $\mathbf{2 5}$ children. Fifteen of 29 had growth hormone insufficiency 0.9-4.9 years after total body irradiation, yet only three of the 15 had received previous cranial irradiation. Five of $\mathbf{3 0}$ had thyroid dysfunction: two with a low thyroxine and raised thyroid stimulating hormone (TSH) concentration and three with a raised TSH and normal thyroxine concentration. Thus the incidence of thyroid dysfunction $(16 \%)$ is much lower than that reported after single fraction total body irradiation (39-59\%). In only two children were abnormalities of the hypothalamicpituitary-adrenal axis demonstrated. The majority of pubertal children assessed $(n=15)$ showed evidence of gonadal damage. All the pubertal girls $(n=5)$ had ovarian failure, although there was evidence of recovery of ovarian function in one girl. All seven boys in late puberty showed evidence of damage to the germinal epithelium, and two of three in early puberty had raised follicle stimulating hormone concentrations. Despite the use of a fractionated total body irradiation regimen, endocrine morbidity is substantial and children undergoing such procedures will require long term endocrine review and management.
\end{abstract}

\section{(Arch Dis Child 1992;67:1107-10)}

Bone marrow transplantation (BMT) is now an established treatment for refractory malignant disease such as acute lymphoblastic leukaemia, either in first remission or after a relapse, and acute myeloblastic leukaemia in first remission. Since the 1960 s, when BMT was almost universally unsuccessful, advances in histocompatibility testing and improvements in supportive care have led to increasing numbers of young adults surviving childhood malignancy. Five year survival rates have increased dramatically from $2 \%$ to $47 \%$ in acute lymphoblastic leukaemia and $2 \%$ to $21 \%$ in acute myeloblastic leukaemia between the decades 1954-63 and 1974-83. ${ }^{1}$

To date most of the work on the endocrine consequences of BMT have been described in an adult population in whom particularly high incidences of gonadal and thyroid dysfunction have been reported. ${ }^{2}$ There is little information on the outcome in children. In the only sizable surveys, single fraction total body irradiation was used in the preparative regimen, ${ }^{3}$ or a population was described in whom either single fraction or fractionated total body irradiation was employed. ${ }^{4}$ It is not clear in the latter study if the prevalence of endocrine complications differ after the two irradiation schedules. ${ }^{4}$ Both studies report a high incidence of growth hormone deficiency, hypogonadism, and thyroid dysfunction. ${ }^{34}$

We have studied the endocrine morbidity after BMT in children from two major UK centres at which the total body irradiation is delivered in multiple fractions rather than a single dose, to assess whether this has a less detrimental endocrine outcome.

\section{Patients and methods}

Thirty one children (17 boys) who received total body irradiation before BMT between 1978 and 1990 at the Royal Hospital for Sick Children, Glasgow, and the Christie Hospital, Manchester were studied. All were in complete remission at the time of endocrine assessment, which was performed between 0.5 and 7.8 years after total body irradiation. The mean age at transplantation was $8 \cdot 1$ (range $2 \cdot 2-15 \cdot 2$ ) years. The primary diagnosis was acute lymphoblastic leukaemia $(n=11)$, acute myeloid leukaemia $(n=15)$, chronic granulocytic leukaemia $(n=3)$, and neuroblastoma $(n=2)$.

Seventeen children underwent allogenic and 14 autologous BMT. The conditioning chemotherapy consisted of cyclophosphamide $60 \mathrm{mg} /$ $\mathrm{kg}$ /day on two consecutive days (in 29 children), or melphalan $140 \mathrm{mg} / \mathrm{m}^{2}$ as a single dose (in the two children with neuroblastoma).

All 31 children received fractionated whole body irradiation, delivered by a $4 \mathrm{MV}$ linear accelerator at a dose rate of $10 \mathrm{cGY} /$ minute. The median dose was 1200 (range 1100-1520) cGy in 6 (3-8) fractions, over 3.5 (2-5) days.

In the treatment of the original disease, all children had received multiagent cytotoxic chemotherapy for varying lengths of time. Children with acute lymphoblastic leukaemia were treated with established Medical Research Council (MRC) protocols. Patients with acute myeloid leukaemia were treated with either the MRC acute myeloid lymphoma protocols or the Bristol Myers protocol. All patients with acute lymphoblastic leukaemia or acute myeloid leukaemia received intrathecal methotrexate, and/or cytosine arabinoside. Patients with chronic granulocytic leukaemia were treated with hydroxyurea with or without busulfan. The patients with neuroblastoma received six courses of OPEC chemotherapy (vincristine, cis-platinum, etoposide, and cyclophosphamide). 
Twenty seven children were transplanted in first remission and four in second remission. Seven children had received radiotherapy before total body irradiation. Four children received cranial irradiation at a dose of $1800-2400 \mathrm{cGy}$ at a median time of 3.7 (range $3 \cdot 2-3 \cdot 8$ ) years before total body irradiation. Two of these four also received testicular irradiation at a dose of 1000 and $600 \mathrm{cGy}$ in six and three fractions respectively over three days. One child received mediastinal irradiation, one orbital irradiation, and one received splenic irradiation.

Only one child developed graft-versus-host disease, which was treated with prednisolone for seven weeks. No child was taking immunosuppressive treatment at the time of endocrine assessment.

In all 31 patients basal concentrations of gonadotrophins (follicle stimulating hormone (FSH) and luteinising hormone), oestradiol or testosterone, thyroxine, and thyroid stimulating hormone (TSH) were measured. In 19 children a basal measurement of prolactin was made.

Twenty nine patients underwent full combined dynamic tests of hypothalamic-pituitary function performed in the fasting state between 0.5 and $7 \cdot 8$ years after BMT. Growth hormone secretion and the pituitary-adrenal axis were assessed by an insulin tolerance test $(0.2 \mathrm{U} / \mathrm{kg}$ human soluble insulin). The gonadotrophin response to an intravenous bolus of gonadotrophin releasing hormone (GnRH) $\left(100 \mu \mathrm{g} / \mathrm{m}^{2}\right.$, to a maximum of $100 \mu \mathrm{g}$ ) and $\mathrm{TSH}$ response to an intravenous bolus $(3 \mu \mathrm{g} / \mathrm{kg}$ to a maximum of $200 \mu \mathrm{g}$ ) of thyrotrophin releasing hormone (TRH) were also assessed.

Pubertal status was assessed clinically by the method of Tanner. ${ }^{5}$ Testicular volumes were estimated by comparison with the Prader orchidometer. $^{6}$ None of the patients was taking corticosteroids or thyroxine at the time of dynamic testing. Sex steroid replacement therapy was discontinued four weeks before endocrine assessment because of the potential effect of exogenous oestrogen on cortisol binding and thyroxine binding globulins.

All hormone measurements were performed using standard radioimmunoassays.

\section{Results}

Twenty five of 31 children had abnormal endocrine function.

\section{GROWTH HORMONE}

Fifteen of 29 (52\%) children had growth hormone insufficiency as defined by a growth hormone response of less than $10 \mu \mathrm{g} / 1(20 \mathrm{mU} / \mathrm{l})$ to an insulin tolerance test, performed a mean time of 2.4 (range $0 \cdot 5-7 \cdot 4$ ) years from total body irradiation. Three of the 15 had received previous cranial irradiation. One child, who had received cranial irradiation in addition to total body irradiation, had normal growth hormone secretion to provocative testing. Nine children are now receiving growth hormone treatment.

THYROID FUNCTION

Thyroid function was assessed between 0.6 and
$7 \cdot 8$ (mean (SD) $3 \cdot 2(1 \cdot 8)$ ) years after total body irradiation. Five of 30 children showed evidence of thyroid dysfunction. Two had primary hypothyroidism (thyroxine $<50 \mathrm{nmol} / 1$, TSH $>6 \mathrm{mU} / \mathrm{l})$ and three compensated thyroid dysfunction (normal thyroxine, TSH concentration $>6 \mathrm{mU} / \mathrm{l}$ ), two of whom showed an exaggerated peak TSH response to TRH. (Normal peak TSH response to TRH between 5-32 $\mathrm{mU} / \mathrm{l}^{7}$ ) All five are receiving thyroxine.

\section{ADRENAL FUNCTION}

Two children had biochemical evidence of an abnormality of the pituitary-adrenal axis with a very low basal cortisol concentration in both ( 50 and $60 \mathrm{nmol} / 1$ respectively) and a borderline low peak cortisol response to insulin hypoglycaemia of $413 \mathrm{nmol} / \mathrm{l}$ in one child (normal $>500 \mathrm{nmol} / \mathrm{l}$ ).

\section{GONADAL FUNCTION}

Gonadal function was studied in all 31 children.

All five girls who were pubertal at the time of gonadal assessment had ovarian failure with raised basal gonadotrophins, exaggerated gonadotrophin responses to $\mathrm{GnRH}$, and low oestradiol concentrations. All five have required sex steroid therapy to induce or maintain progress in puberty, to avoid symptoms of oestrogen deficiency, and to reduce the risks of osteoporosis. One girl, pubertal at the time of total body irradiation, developed ovarian failure after the procedure but has since shown evidence of recovery of ovarian function two years after the total body irradiation. Although the adequacy of ovarian function could not be assessed comprehensively in the children who were prepubertal at the time of the study, the older girls of peripubertal age showed evidence of ovarian damage with raised basal gonadotrophins and low oestradiol concentrations.

All seven boys in late puberty at the time of endocrine assessment had a raised basal FSH concentration indicative of damage to the germinal epithelium, and in six of the seven the testicular volumes were small for pubertal stage. One had had previous testicular irradiation. Two of the three boys in early puberty had a raised FSH concentration but no firm conclusions could be drawn about their testicular size at this pubertal stage (G2, PH2). One of the three had received previous testicular irradation.

No boy had overt Leydig cell failure. Those boys in late puberty had normal testosterone concentrations, although four of seven had raised basal luteinising hormone concentrations. One of the three boys in early puberty had a raised luteinising hormone. Seven boys were still prepubertal at the time of the study and therefore the adequacy of testicular function could not be assessed. However although all had normal testosterone and luteinising hormone concentrations, the FSH was raised in three of the seven boys.

\section{PROLACTIN}

Only one of 19 children (5\%) had a prolactin concentration $(422 \mathrm{mU} / \mathrm{l})$ above the normal range of $350 \mathrm{mU} / 1$. 


\section{Discussion}

The radiobiological effect of irradiation on normal tissues is related not only to the total dose of radiation delivered, but also to the dose per fraction and is inversely related to the overall treatment time. ${ }^{89}$ It would be anticipated therefore that fractionated total body irradiation would cause less endocrine deficit than single fraction total body irradiation. None the less, in this study which includes only patients receiving fractionated total body irradiation we have shown that endocrine morbidity is considerable. Confounding variables were restricted to a minimum in that only four of the $\mathbf{3 1}$ children had received previous cranial irradiation, only one child had experienced a previous episode of graft-versus-host disease, and no child was currently on steroids or chemotherapy.

Despite a total total body irradiation dose of only 1100-1520 cGy to the hypothalamicpituitary axis, $52 \%(15 / 29)$ of children were growth hormone insufficient after total body irradiation (mean interval 2.4 years), yet only three of these had previous cranial irradiation. Sanders et al described a similar incidence of growth hormone insufficiency in children one to 13 years after receiving total body irradiation as a single dose (920-1000 cGy) or fractionated regimens of 1200-1575 cGy over 6-7 days but did not distinguish between the impact of these two schedules. ${ }^{410}$ It is likely that the prevalence of growth hormone insuffiency will increase with time from total body irradiation ${ }^{11} 12$ and thus long term studies will be required to appreciate the full impact of fractionated total body irradiation on growth hormone secretion. Adults receiving similar doses of fractionated total body irradiation, however, do not develop growth hormone insufficiency when assessed at a similar time from irradiation, ${ }^{2}$ suggesting that growth hormone secretion in the prepubertal and early pubertal child is more vulnerable to the effects of hypothalamic-pituitary irradiation than in the adult.

In this study we have concentrated only on endocrine deficit after total body irradiation and not the effect of fractionated total body irradiation on growth. Little is known about the effect of fractionated total body irradiation on cartilage growth and the resultant effect on body proportions in those with normal and subnormal growth hormone secretion. More longitudinal data were required before these effects can be meaningfully assessed.

The incidence of thyroid dysfunction (16\%) after fractionated total body irradiation is much lower than that reported after single fraction total body irradiation (39-59\%). ${ }^{34}$ The incidence of thyroid dysfunction may vary with time in that recovery of thyroid function has been observed in patients with documented thyroid dysfuction after total body irradiation. ${ }^{13}$ Radiation appears to be the major, if not sole cause of subsequent thyroid dysfunction as BMT without irradiation is not associated with thyroid dysfunction. ${ }^{14}$ Furthermore, preirradiation treatment with multiple chemotherapeutic agents does not further increase the incidence of thyroid dysfunction. ${ }^{14}$
We found a low incidence of hypothalamicpituitary-adrenal dysfunction (7\%) after fractionated total body irradiation compared with the incidence of $24 \%$ reported by Sanders et al. ${ }^{4}$ The adrenal gland is relatively radioresistant and impairment of corticotrophin secretion is not seen in children receiving $1800-2400 \mathrm{cGy}$ cranial irradiation prophylactically for acute lymphoblastic leukaemia. ${ }^{15}$ The high incidence in the report by Saunders et al may be due to the suppression of the hypothalamic-pituitaryadrenal axis associated with the previous use of high dose steroids to treat either the undelying condition or graft-versus-host disease. ${ }^{4}$ Moreover, use of the metyrapone test, which has been shown to have a significant failure rate in normal individuals, ${ }^{16}$ may also explain the high incidence of cortisol deficiency previously reported. ${ }^{4}$

Severe gonadal damage is common after total body irradiation. All girls of pubertal age at time of assessment had ovarian failure irrespective of their pubertal status at the time of irradiation. Although the ovary is less sensitive to the effects of irradiation than the testis, the $\mathrm{LD}_{50}$ (the radiation dose causing death to $50 \%$ of oocytes) has been estimated to be less than $400 \mathrm{cGy} .{ }^{17} \mathrm{~A}$ similar incidence of ovarian failure is reported after single fraction total body irradiation. $^{3}$

Most of the boys exhibited severe damage to the germinal epithelium yet none had overt Leydig cell failure. There is evidence to show that the germinal epithelium of the testis is more vulnerable to the effects of both cytotoxic chemotherapy and irradiation than either the ovary or the Leydig cell. ${ }^{18}$ Therefore the universal finding of damage to the germinal epithelium would be anticipated after both gonadotoxic chemotherapy and irradiation. Germ cell dysfunction induced by similar conditioning chemotherapy and total body irradiation administered as a single fraction appears to be amenable to recovery as demonstrated by a return to normal of the previously raised FSH concentration with time, although semen analysis was not performed. ${ }^{19}$ Animal evidence suggests that irradiation administered as a fractionated schedule is more deleterious to spermatogenesis than the same total irradiation dose in a single fraction. Recovery of spermatogenesis has, however, been described in adults who received fractionated total body irradiation. ${ }^{2}$

Unlike the testis, fractionated irradiation is less damaging to the ovary than single fraction irradiation, ${ }^{20}$ although such differences may be lost at the doses employed in total body irradiation. In addition, all the girls in this study received a combination of gonadotoxic chemotherapy and fractionated irradiation, both of which may contribute to the development of ovarian failure. Recovery of ovarian function with time has been reported after both fractionated and single fraction total body irradiation, ${ }^{4}$ and in our study one girl showed evidence of recovery of ovarian function two year after total body irradiation.

In conclusion, $80 \%$ of children had evidence of endocrine dysfunction a mean of 2.4 years after total body irradiation administered in a 
fractionated schedule. In such children there is a wide range of endocrine disturbance affecting the hypothalamic-pituitary axis, the thyroid, and the gonad. Close and early involvement of an endocrinologist in their management is mandatory.

We thank the Leukaemia Research Fund and Serono laboratories (UK) Ltd for financial support.

1 Birch JM, Marsden HB, Morris Jones PH, Pearson D, Blair V. Improvements in survival from childhood cancer: results of a population based survey over 30 years. $B M \mathcal{J}$ 1988;296:1372-6.

2 Littley MD, Shalet SM, Morgenstern GR, Deakin DP. Endocrine and reproductive dysfunction following fractionated total body irradiation in adults. $Q$ I Med 1991;78:265-74.

3 Leiper AD, Stanhope R, Lau T, et al. The effect of total body irradiation and marrow transplantation during childhood and adolescence on growth and endocrine function. $\mathrm{Br}$ and Haematol 1987;67:419-26.

4 Sanders JE, Pritchard S, Mahoney P, et al. Growth and development following marrow transplantation for development following marrow
leukaemia. Blood $1986 ; 68: 1129-35$.

5 Tanner JM. Growth at adolescence. Oxford: Blackwell Scientific, 1962

6 Zachmann M, Prader A, Kind HP, Hafliger H, Budliger $H$. Testicular volumes during adolescence. Cross sectional and longitudinal studies. Helvetica Pediatrica Acta 1974;29: 61-72.

7 Shalet SM, Beardwell CG, Twomey JA, Morris Jones PH, Pearson D. Endocrine function following the treatment of acute leukaemia in childhood. $\mathcal{f}$ Pediatr 1977;90:920-3.
8 Peters L. Discussion: the radiobiological bases of TBI. Int f Radiat Oncol Biol Phys 1980;6:785-7.

9 Hendry JH. Response of human organs to single (or fractionated equivalent) doses of irradiation. Int $\mathcal{Y}$ Radiat Biol 1989;56:691-700.

10 Sanders JE, Buckner CD, Sullivan KM, et al. Growth and development in children after bone marrow transplantation. Horm Res 1988;30:93-7.

11 Borgstrom B, Bolme P. Growth and growth hormone in children after bone marrow transplantation. Horm Res 1988;30:98-100.

12 Clayton PE, Shalet SM. Dose dependency of time of onset of radiation-induced growth hormone deficiency. $\mathcal{F}$ Pediatr 1991:118:226-8.

13 Katsanis E, Shapiro RS, Robison LL, et al. Thyroid dysfunction following bone marrow transplantation: longterm follow-up of 80 pediatric patients. Bone Marrow Transplant 1990;5:335-40.

14 Sklar CA, Kim TH, Ramsay NKC. Thyroid dysfunction among long-term survivors of bone marrow transplantation. Am F Med 1982;73:688-94.

15 Wallace WHB, Crowne EC, Shalet SM, Moore C, Gibson S, White A. Episodic ACTH and cortisol secretion after cranial irradiation (CI) in childhood. Horm Res 1990 33 (suppl 3): 172.

16 Best JD, Alford FP, Donald RA. Evaluation of the three hour metyrapone test in adults. Clin Endocrinol $(O x f)$ 1980;13: 69-76.

17 Wallace WHB, Shalet SM, Hendry JH, Morris-Jones PH Gattamaneni HR. Ovarian failure following total abdominal irradiation in childhood: the radiosensitivity of the human irradiation in childhood: the radiosen

18 Shalet SM. Gonadal function following radiation and cytotoxic chemotherapy in childhood. In: Brandis $M$, Frick $P$, Kochsiek K, et al, eds. Advances in internal medicine and pediatrics. Vol 58. Berlin: Springer-Verlag, 1989:1-21.

19 Sklar CA, Kim TH, Ramsay NKC. Testicular function following bone marrow transplantation performed during or after puberty. Cancer 1984;53:1498-501.

20 Ash $\mathrm{P}$. The influence of radiation on fertility in man. $\mathrm{Br} \mathcal{F}$ 